\title{
Iridoschisis and keratoconus in a patient with severe allergic eye disease and compulsive eye rubbing: a case report
}

\author{
Imran H. Yusuf and John F. Salmon *
}

\begin{abstract}
Background: Iridoschisis is a rare disorder characterized by splitting of the anterior and posterior iris stroma, resulting in disintegrated iris fibrils which float freely in the anterior chamber. We report an exceptional case of bilateral iridoschisis occurring in conjunction with keratoconus and severe allergic eye disease.

Case presentation: A 24-year-old white man had had periocular contact dermatitis and allergic eye disease from the age of 3 years. He was allergic to grass, animal hair, and pollen and worked grooming horses. He compulsively rubbed his eyes. There was no history of previous blunt trauma to either eye. There were signs of bilateral iridoschisis and keratoconus with allergic conjunctivitis, all of which were more severe in his right eye. An open drainage angle was identified bilaterally on gonioscopy, excluding primary angle closure. There was no evidence of glaucoma in either eye.

Conclusions: There are two previous cases reporting the combination of iridoschisis and keratoconus, but no clear common etiology has been identified. In this case there was no evidence of angle closure but there were signs of allergic conjunctivitis. This amalgamation of signs might be explained on the basis of habitual eye rubbing. Treating the allergic eye disease has attenuated this behavior.
\end{abstract}

Keywords: Iridoschisis, Keratoconus, Allergic eye disease, Eye rubbing, Open drainage angle

\section{Background}

Iridoschisis is a rare disorder characterized by localized cleavage of the anterior and posterior iris stroma [1]. The anterior leaf of the iris is atrophic and has disintegrated into fibrils, which float freely in the anterior chamber. A clear pathogenesis of iridoschisis has not been identified, although partial or complete angle closure in association with reduced axial length and shallow anterior chamber depth is commonly found [2,3]. We report an exceptional case of bilateral iridoschisis, keratoconus, and severe allergic eye disease in a patient with an open drainage angle and a deep anterior chamber in both eyes. This case suggests the intriguing possibility that his ophthalmic pathology may be explained on the basis of habitual eye rubbing.

* Correspondence: john.salmon@ouh.nhs.uk

The Oxford Eye Hospital, West Wing, John Radcliffe Hospital, Headley Way, Headington, Oxford OX3 9DU, UK

\section{Case presentation}

A 24-year-old right-handed white man presented with a 21-year history of compulsive eye rubbing secondary to periocular atopic dermatitis and allergic conjunctivitis. He was allergic to animals, grass, and pollen and worked grooming horses. Topical emedastine $0.05 \%$ drops, periocular $1 \%$ hydrocortisone, and oral antihistamines were used to control his symptoms. His general health was excellent.

His corrected visual acuity was 6/7.4 right eye $(\mathrm{OD} ;-5.25 /-6.50 \times 30)$ and $6 / 9$ left eye $(\mathrm{OS} ;-2.00 /-$ $4.00 \times 95)$ with irregular astigmatism. There was evidence of periocular dermatitis with mild scaling and lichenification. Papillae were present on the upper tarsal conjunctivae, typical of allergic conjunctivitis. There were no other ocular signs of atopic disease. His intraocular pressure was $15 \mathrm{mmHg}$ for both eyes (OU). Bilateral, asymmetrical inferior iridoschisis was present without corectopia or ectropion uveae (Fig. 1a, b). His anterior chamber was deep. Gonioscopy identified an 

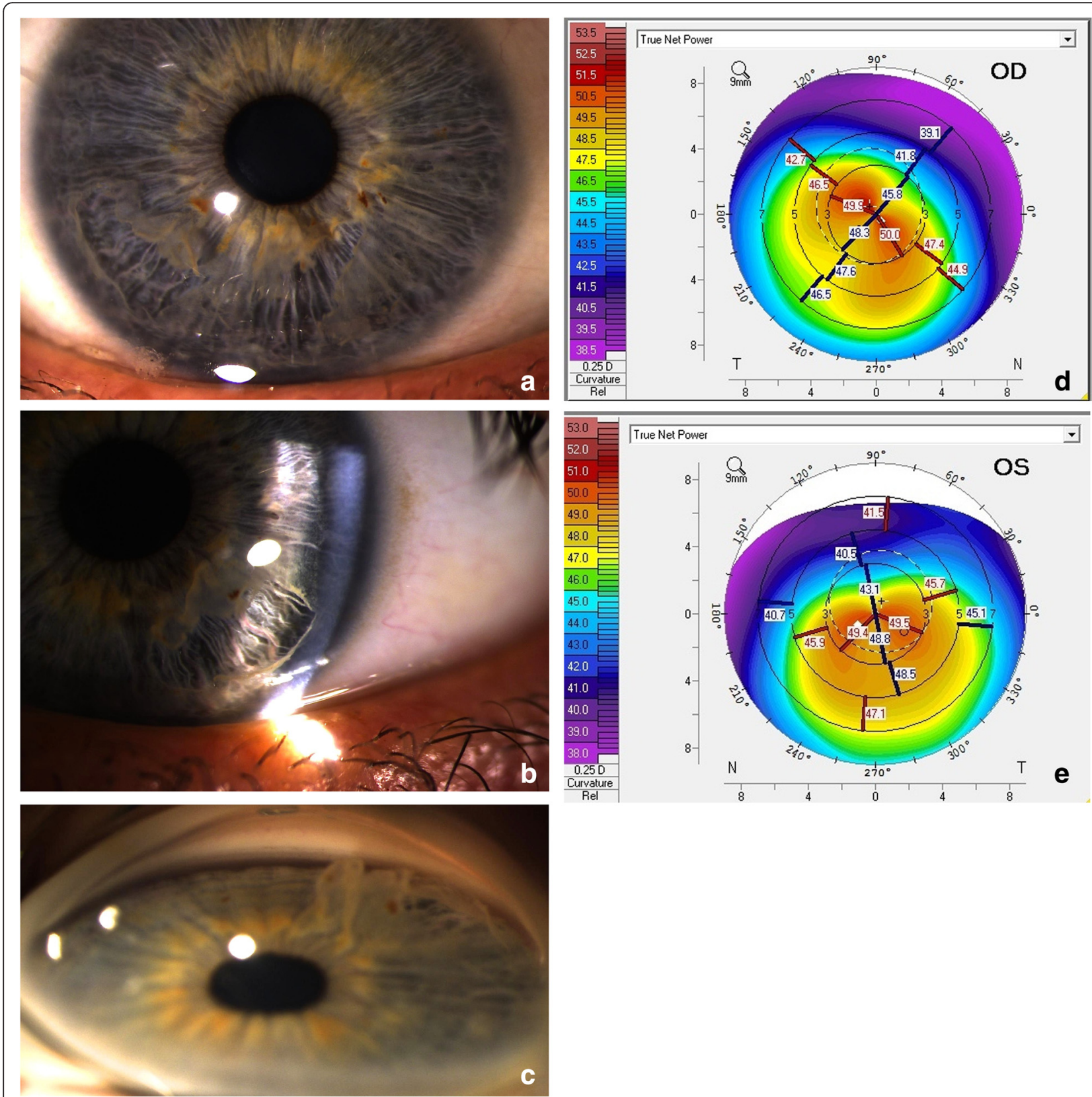

Fig. 1 Anterior segment photograph of right (a) and left eyes (b) demonstrating asymmetrical inferior iridoschisis worse on the right, maximal in the inferotemporal quadrant. Anterior iris stroma can be identified protruding into the deep anterior chamber. Gonioscopy reveals an open drainage angle with a ciliary body band visible in the inferior drainage angle of the left eye (c). Note the protrusion of the anterior iris stroma into the anterior chamber on gonioscopy. Oculus Pentacam corneal topography (anterior surface) demonstrates bilateral keratoconus, worse on the right (d) than the left (e) with apical decentration. Central corneal thickness was 449 microns right eye and 429 microns left eye. $O D$ right eye, OS left eye

open drainage angle allowing the ciliary body to be visualized through 360 degrees OU (Fig. 1c). Scheimpflug imaging measured an anterior chamber depth of $3.48 \mathrm{~mm}$ OD and $3.45 \mathrm{~mm}$ OS. Corneal topography documented bilateral keratoconus (Fig. 1d, e). A posterior segment examination revealed a healthy optic disc and macula.
There was no family history of keratoconus or iridoschisis.

\section{Discussion}

There are only two previous cases reporting the rare combination of iridoschisis and keratoconus $[4,5]$. The 
authors of these reports struggled to identify a unifying mechanism of keratoconus and iridoschisis, suggesting the unlikely hypotheses of a potential genetic association or common embryological derivation of involved tissues. Eye rubbing was not commented on specifically in such reports; it was not known to be strongly associated with keratoconus at the time. This report suggests, for the reasons outlined below, that eye rubbing may represent the common origin of coexistent keratoconus and iridoschisis.

Over the past 60 years since the term iridoschisis was first proposed by Loewenstein and Foster [6], numerous case reports and several case series have attempted to identify a common pathogenesis of iridoschisis from a variety of reported etiologies. Primary angle closure is strongly associated with iridoschisis [1, 7-9], and may be associated with glaucoma in approximately twothirds of patients. One series identified complete or partial angle closure in 12 consecutive patients with iridoschisis leading to the conclusion that symptomatic or subclinical intraocular pressure spikes were contributory to its development [1]. The association of iridoschisis and plateau iris syndrome has recently been described in a young patient [10]. Gonioscopy and Scheimpflug imaging excluded primary angle closure in our patient. Trauma was considered a contributory factor in the development of iridoschisis by Loewenstein 1948 [11] and was supported by a subsequent report by Salmon [2]. In a similar fashion to primary angle closure, post-traumatic intraocular pressure peaks may result in shearing along dilator fibers with consequent splitting of the iris stroma $[9,11]$. Subluxation of the crystalline lens resulting in adjacent sectoral angle closure and iridoschisis has been reported [12], with mechanical trauma from a mobile crystalline lens suggested to be contributing to its development. The possibility of intraocular pressure peaks in the setting of lens subluxation due to a secondary angle closure mechanism suggests similarities in pathogenesis in patients with primary angle closure. In summary, peaks in intraocular pressure in association with iris trauma are the most consistent themes in previous reports of iridoschisis. Chronic eye rubbing combines these insults to the iris: mechanical trauma and intraocular pressure spikes, which over years may conceivably have contributed to the development of iridoschisis in this case.

Ocular manifestations of atopic dermatitis include periocular dermatitis, atopic keratoconjunctivitis, atopic cataract, retinal detachment, and rarely, cicatricial lid disease (symblepharon, entropion, and so on).

Keratoconus is strongly associated with habitual eye rubbing. Multivariate analysis identified eye rubbing as the only statistically significant risk factor for keratoconus, after adjustment for atopy, among all commonly perceived risk factors [13]. Our patient was right hand dominant and the keratoconus was more pronounced in his right eye, compared to the left. A significant relationship between severe eye rubbing and keratoconus on the side of hand dominance has previously been demonstrated [14].

\section{Conclusions}

In conclusion, this patient had keratoconus, iridoschisis, and severe allergic eye disease. This amalgamation of signs, particularly obvious in his right eye, might be explained on the basis of habitual eye rubbing. His behavior has been attenuated by treating the allergic eye disease.

\section{Acknowledgments \\ Thanks to Dr Jon Brett at the Oxford Eye Hospital for taking the anterior segment photographs presented in this manuscript.}

\section{Authors' contributions}

Both IHY and JFS have: 1) made substantial contributions to conception and design, or acquisition of data, or analysis and interpretation of data; 2) have been involved in drafting the manuscript or revising it critically for important intellectual content; 3 ) have given final approval of the version to be published; and 4) agree to be accountable for all aspects of the work in ensuring that questions related to the accuracy or integrity of any part of the work are appropriately investigated and resolved.

\section{Competing interests}

The authors declare that they have no competing interest.

\section{Consent}

Written informed consent was obtained from the patient for publication of this case report and accompanying images. A copy of the written consent is available for review by the Editor-in-Chief of this journal.

Received: 22 October 2015 Accepted: 26 April 2016

Published online: 25 May 2016

\section{References}

1. Salmon JF, Murray AD. The association of iridoschisis and primary angleclosure glaucoma. Eye. 1992;6(Pt 3):267-72. doi:10.1038/eye.1992.50.

2. Salmon JF. The association of iridoschisis and angle-recession glaucoma. Am J Ophthalmol. 1992;114(6):766-7.

3. Rodrigues MC, Spaeth GL, Krachmer JH, Laibson PR. Iridoschisis associated with glaucoma and bullous keratopathy. Am J Ophthalmol. 1983;95(1): 73-81.

4. Eiferman RA, Law M, Lane L. Iridoschisis and keratoconus. Cornea. 1994; 13(1):78-9.

5. Krohn DL, Garrett EE. Iridoschisis and keratoconus; report of case in a twenty-year-old man. Arch Ophthalmol. 1954;52(3):426-32.

6. Loewenstein A, Foster J. Iridoschisis with multiple rupture of stromal threads. Br J Ophthalmol. 1945;29(6):277-82.

7. Romano A, Treister G, Barishak R, Stein R. Iridoschisis and angle-closure glaucoma. Int J Ophthalmol. 1972;164(3):199-207.

8. Shima C, Otori Y, Miki A, Tano Y. A case of iridoschisis associated with plateau iris configuration. Jpn J Ophthalmol. 2007:51(5):390-1. doi:10.1007/ s10384-007-0454-1.

9. Gogaki E, Tsolaki F, Tiganita S, Skatharoudi C, Balatsoukas D. Iridoschisis: case report and review of the literature. Clin Ophthalmol. 2011;5:381-4. doi:10.2147/opth.s17269.

10. Chapman $\mathrm{KO}$, Demetriades $\mathrm{AM}$. Juvenile iridoschisis and incomplete plateau iris configuration. J Glaucoma. 2015;24(5):e142-4. doi:10.1097/ijg.0000000000000161.

11. Loewenstein A, Foster J, Sledge SK. A further case of iridoschisis. Br J Ophthalmol. 1948;32(3):129-34.

12. Agrawal S, Agrawal J, Agrawal TP. Iridoschisis associated with lens subluxation. J Cataract Refract Surg. 2004;30(9):1825. doi:10.1016/j.jcrs.2004.06.065. 
13. Bawazeer AM, Hodge WG, Lorimer B. Atopy and keratoconus: a multivariate analysis. Br J Ophthalmol. 2000;84(8):834-6.

14. McMonnies CW, Boneham GC. Keratoconus, allergy, itch, eye-rubbing and hand-dominance. Clin Exp Optom. 2003;86(6):376-84.

Submit your next manuscript to BioMed Central and we will help you at every step:

- We accept pre-submission inquiries

- Our selector tool helps you to find the most relevant journal

- We provide round the clock customer support

- Convenient online submission

- Thorough peer review

- Inclusion in PubMed and all major indexing services

- Maximum visibility for your research

Submit your manuscript at www.biomedcentral.com/submit 